\title{
Intuitionistic Trapezoidal Fuzzy Multiple Criteria Group Decision Making Method Based on Binary Relation
}

\author{
Liyuan Zhang, ${ }^{1}$ Tao Li, ${ }^{2}$ and $\mathrm{Xuanhua} \mathrm{Xu}^{3}$ \\ ${ }^{1}$ School of Business, Shandong University of Technology, Zibo, Shandong 255049, China \\ ${ }^{2}$ School of Science, Shandong University of Technology, Zibo, Shandong 255049, China \\ ${ }^{3}$ School of Business, Central South University, Changsha, Hunan 410083, China \\ Correspondence should be addressed to Liyuan Zhang; zhangliyuancsu@163.com
}

Received 23 April 2014; Accepted 13 July 2014; Published 22 July 2014

Academic Editor: Wei-Chiang Hong

Copyright (C) 2014 Liyuan Zhang et al. This is an open access article distributed under the Creative Commons Attribution License, which permits unrestricted use, distribution, and reproduction in any medium, provided the original work is properly cited.

\begin{abstract}
The aim of this paper is to develop a methodology for intuitionistic trapezoidal fuzzy multiple criteria group decision making problems based on binary relation. Firstly, the similarity measure between two vectors based on binary relation is defined, which can be utilized to aggregate preference information. Some desirable properties of the similarity measure based on fuzzy binary relation are also studied. Then, a methodology for fuzzy multiple criteria group decision making is proposed, in which the criteria values are in the terms of intuitionistic trapezoidal fuzzy numbers (ITFNs). Simple and exact formulas are also proposed to determine the vector of the aggregation and group set. According to the weighted expected values of group set, it is easy to rank the alternatives and select the best one. Finally, we apply the proposed method and the Cosine similarity measure method to a numerical example; the numerical results show that our method is effective and practical.
\end{abstract}

\section{Introduction}

With the development of internet technology, multiple criteria group decision making (MCGDM) becomes an important part of modern decision science. It has been extensively applied to various areas, such as society, economics, management, military, and engineering technology. Since Bellman and Zadeh [1] initially proposed the fuzzy decision making model based on the fuzzy mathematical theory, many achievements have been made in literature on fuzzy multiple criteria group decision making (FMCDM) problems [2]. Some related fuzzy decision making methods were proposed in the references, for example, weighted aggregation operators [3], ordered weighted aggregation operators [4], weighted geometric aggregation operators [5], TOPSIS method [6], analytic hierarchy process method [7], grey relational analysis method [8], similarity measures [9], and so on. Similarity measure is an important tool to determine the degree of similarity between two fuzzy uncertain objects, which has successfully applied in FMCDM problem. Many different similarity measures for FMCDM problems have been investigated in the literature [10-14]. Based on the extension of the Hamming distance on fuzzy sets, Szmidt and Kacprzyk [15] introduced the Hamming distance between two intuitionistic fuzzy sets (IFSs) to define the similarity measure for IFSs and applied the method to group decision making. On the other hand, $\mathrm{Xu}$ [16] developed some similarity measures of IFSs based on the geometric distance and defined the notions of positive ideal IFS and negative ideal IFS, which can be applied to multiple attribute decision making under intuitionistic fuzzy environment. But as an effective method to solve FMCDM problem, it is rarely applied in intuitionistic trapezoidal fuzzy group decision making.

Nehi and Maleki [17] firstly introduced the ITFN and the operation law of ITFNs. Because the ITFN is intuitive and easy to use, many researchers used it to express the preference information of the experts and the criteria values. Ye [18] used the ITFN to express the decision information. Based on the expected values for ITFNs, he presented a handling method for intuitionistic trapezoidal fuzzy multiple attribute decision making. In [19], Ye used the vector similarity measures between two ITFNs to rank the alternative, through the weighted similarity measures between each alternative and ideal alternative, and it was easy to select the best one. 
However, the above methods are without considering the binary relation between decision criteria. In the complex reallife case, it has complex binary relation between each criterion. For example, in emergency resource allocation decision making problem, food and water (i.e., criteria) have "proportion" relationship (i.e., binary relation). In this case, some traditional FMCGDM methods are unsuitable to solve this problem. So in this paper, we introduce a new method for FMCGDM based on binary relation of criteria, with intuitionistic trapezoidal fuzzy information. The rest of this paper is arranged as follows: in the next section, we firstly introduce some basic notions and preliminary definitions of fuzzy numbers and intuitionistic trapezoidal fuzzy numbers; Section 3 contains a new degree of similarity between two vectors based on binary relation; in Section 4, the clustering algorithm based on binary relation similarity measure is given, and we use an example to compare with the other two clustering algorithms; we propose a new FMCGDM method based on binary relation in Section 5; then, we give an illustrative example to authenticate the method and discuss the result of numerical example in Section 6; the results of the Cosine similarity measure are also given for comparison; the paper ends with conclusion in Section 7.

\section{Preliminaries}

We consider the following well-known description of a fuzzy number $\alpha$.

Definition 1 (Dubois and Prade [20]). Let $\alpha$ be a fuzzy number in the set of real number $R$; its membership function is defined as follows:

$$
\mu_{\alpha}(x)= \begin{cases}0, & x<a_{1}, \\ f_{\alpha}(x), & a_{1} \leq x \leq a_{2}, \\ 1, & a_{2} \leq x \leq a_{3}, \\ g_{\alpha}(x), & a_{3} \leq x \leq a_{4}, \\ 0, & a_{4}<x,\end{cases}
$$

where $a_{1}, a_{2}, a_{3}, a_{4} \in R, f_{\alpha}:\left[a_{1}, a_{2}\right] \rightarrow[0,1]$ is a nondecreasing continuous function, $f_{\alpha}\left(a_{1}\right)=0, f_{\alpha}\left(a_{2}\right)=1$, called the left side of fuzzy number $\alpha$ and $g_{\alpha}:\left[a_{3}, a_{4}\right] \rightarrow[0,1]$ is a nonincreasing continuous function, $g_{\alpha}\left(a_{3}\right)=1, g_{\alpha}\left(a_{4}\right)=0$, called the right side of fuzzy number $\alpha$.

The intuitionistic trapezoidal fuzzy number, which is expressed by a membership function and nonmembership function, is shown as follows.

Definition 2 (Ye [19]). An ITFN $\alpha$ with parameters $b_{1} \leq$ $a_{1} \leq b_{2} \leq a_{2} \leq b_{3} \leq a_{3} \leq a_{4} \leq b_{4}$ is denoted as $\alpha=$ $\left\langle\left(a_{1}, a_{2}, a_{3}, a_{4}\right),\left(b_{1}, b_{2}, b_{3}, b_{4}\right)\right\rangle$ in the set of real number $R$.
In this case, its membership function and nonmembership function can be given as follows:

$$
\begin{aligned}
& \mu_{\alpha}(x)= \begin{cases}0, & x<a_{1}, \\
\frac{x-a_{1}}{a_{2}-a_{1}}, & a_{1} \leq x \leq a_{2}, \\
1, & a_{2} \leq x \leq a_{3}, \\
\frac{x-a_{4}}{a_{3}-a_{4}}, & a_{3} \leq x \leq a_{4}, \\
0, & a_{4}<x,\end{cases} \\
& v_{\alpha}(x)= \begin{cases}0, & x<b_{1}, \\
\frac{x-b_{1}}{b_{1}-b_{2}}, & b_{1} \leq x \leq b_{2}, \\
1, & b_{2} \leq x \leq b_{3}, \\
\frac{x-b_{3}}{b_{4}-b_{3}}, & b_{3} \leq x \leq b_{4}, \\
0, & b_{4}<x .\end{cases}
\end{aligned}
$$

The following properties for ITFN can be seen in [17]. Let $\alpha_{1}=\left\langle\left(a_{11}, a_{12}, a_{13}, a_{14}\right),\left(b_{11}, b_{12}, b_{13}, b_{14}\right)\right\rangle$ and $\alpha_{2}=\left\langle\left(a_{21}\right.\right.$, $\left.\left.a_{22}, a_{23}, a_{24}\right),\left(b_{21}, b_{22}, b_{23}, b_{24}\right)\right\rangle$ be two ITFNs and let $\gamma$ be a positive scalar number. Then,

$$
\begin{array}{r}
\alpha_{1}+\alpha_{2}=\left\langle\left(a_{11}+a_{21}, a_{12}+a_{22}, a_{13}+a_{23}, a_{14}+a_{24}\right),\right. \\
\left.\left(b_{11}+b_{21}, b_{12}+b_{22}, b_{13}+b_{23}, b_{14}+b_{24}\right)\right\rangle, \\
\gamma \alpha_{1}=\left\langle\left(\gamma a_{11}, \gamma a_{12}, \gamma a_{13}, \gamma a_{14}\right),\left(\gamma b_{11}, \gamma b_{12}, \gamma b_{13}, \gamma b_{14}\right)\right\rangle .
\end{array}
$$

Definition 3 (Grzegrorzewski [21]). Let $\alpha=\left\langle\left(a_{1}, a_{2}, a_{3}, a_{4}\right)\right.$, $\left.\left(b_{1}, b_{2}, b_{3}, b_{4}\right)\right\rangle$ be an ITFN in the set of real number $R$; then the expected value of $\alpha$ is defined by

$$
\mathrm{EV}(\alpha)=\frac{E_{*}(\alpha)+E^{*}(\alpha)}{2},
$$

where

$$
\begin{aligned}
& E_{*}(\alpha)=\frac{b_{1}+a_{2}}{2}+\frac{1}{2} \int_{b_{1}}^{b_{2}} h_{\alpha}(x) d x-\frac{1}{2} \int_{a_{1}}^{a_{2}} f_{\alpha}(x) d x, \\
& E^{*}(\alpha)=\frac{b_{4}+a_{3}}{2}+\frac{1}{2} \int_{a_{3}}^{a_{4}} g_{\alpha}(x) d x-\frac{1}{2} \int_{b_{3}}^{b_{4}} k_{\alpha}(x) d x .
\end{aligned}
$$

Therefore, the expected value of ITFNs based on the expected value of IFN can be obtained as the following theorem.

Theorem 4 (Ye [19]). Let $\alpha=\left\langle\left(a_{1}, a_{2}, a_{3}, a_{4}\right),\left(b_{1}, b_{2}, b_{3}, b_{4}\right)\right\rangle$ be an ITFN in the set of real number $R$. Thus, when $f_{\alpha}(x)=$ $\left(x-a_{1}\right) /\left(a_{2}-a_{1}\right), g_{\alpha}(x)=\left(x-a_{4}\right) /\left(a_{3}-a_{4}\right), h_{\alpha}(x)=(x-$ $\left.b_{1}\right) /\left(b_{2}-b_{1}\right), k_{\alpha}(x)=\left(x-b_{3}\right) /\left(b_{4}-b_{3}\right)$, and $a_{1}, a_{2}, a_{3}, a_{4}, b_{1}$, $b_{2}, b_{3}, b_{4} \in R$, its expected value is obtained by

$$
E V(\alpha)=\frac{1}{8}\left(a_{1}+a_{2}+a_{3}+a_{4}+b_{1}+b_{2}+b_{3}+b_{4}\right) .
$$




\section{The New Similarity Based on Binary Relation on Finite Set}

Firstly, we introduce the definition of vector similarity measure.

Definition 5. Let $X=\left(x_{1}, x_{2}, \ldots, x_{n}\right)$ and $Y=\left(y_{1}, y_{2}, \ldots, y_{n}\right)$ be two vectors of length $n$, where all the coordinates are positive; if $S(X, Y)$ satisfies the following properties, $S(X, Y)$ is called a similarity measure between $X$ and $Y$ :

(P1) $0 \leq S(X, Y) \leq 1$;

(P2) $\quad S(X, Y)=S(Y, X)$;

(P3) if $X=Y$, that is, $x_{i}=y_{i}$, for $i=1,2, \ldots, n$,

$$
S(X, Y)=1 \text {. }
$$

Let $Q$ be a binary relation on a finite vector $X$. This relation is defined by its membership function $Q\left(x_{1}, x_{2}\right)$, which maps a direct product $X \times X$ into the unit interval $[0,1]$. For $1 \leq i, j \leq n, M(Q)=\left(m_{i j}\right)_{n \times n}$ is the relation matrix; if and only if $\left(x_{i}, x_{j}\right) \in Q, m_{i j}=1$, or else $m_{i j}=0$. Obviously, $M(Q)$ is a $0-1$ matrix. For example, in emergency resource allocation decision making problem, food and water (i.e., criteria) have "proportion" relationship (i.e., binary relation); the preference vector of the alternative is $V=\left(v_{1}, v_{2}\right)$; $Q$ is defined by its membership function $Q\left(v_{1}, v_{2}\right)$.

Let $M^{1}(Q)$ and $M^{2}(Q)$ be two 0-1 matrixes; the following equation holds true:

$$
M^{1}(Q)+M^{2}(Q)=\left(\operatorname{Max}\left(m_{i j}^{1}, m_{i j}^{2}\right)\right)_{n \times n} .
$$

Definition 6. For the binary relation $Q$, the relation matrixes of the two vectors $X$ and $Y$ are $M$ and $N$, respectively. By using the norm of the matrix, the similarity measure between two vectors is defined as follows:

$$
r(X, Y)=\frac{1}{\sqrt{n}} \frac{\|M+N\|_{2}}{\|M\|_{\infty}+\|N\|_{\infty}},
$$

where $\|M\|_{2}=\left(\rho\left(M^{T} M\right)\right)^{1 / 2},\|M\|_{\infty}=\max _{1 \leq i \leq n} \sum_{j=1}^{n}\left|m_{i j}\right|$, and $\rho\left(M^{T} M\right)$ is the spectral radius of $M^{T} M$, which is the maxis of characteristic value of the matrix $M^{T} M$.

Theorem 7. The similarity measure in Definition 6 satisfies the following properties:

$$
\begin{aligned}
& \text { (P1) } 0 \leq r_{i j}\left(X_{i}, X_{j}\right) \leq 1 ; \\
& (P 2) \quad r_{i i}\left(X_{i}, X_{i}\right)=1 .
\end{aligned}
$$

Proof. (P1) As $\left\|M_{i}+M_{j}\right\|_{2} \geq 0,\left\|M_{i}\right\|_{\infty} \geq 0$, and $\left\|M_{j}\right\|_{\infty} \geq 0$, it is easy to see that $r_{i j}\left(X_{i}, X_{j}\right) \geq 0$. Thus, we only need to prove $r_{i j}\left(X_{i}, X_{j}\right) \leq 1$. By using the property of the norm of the 0-1 matrix,

$$
\|M\|_{2}=\sqrt{n}\|M\|_{\infty}, \quad\left\|M_{i}+M_{j}\right\|_{2} \leq\left\|M_{i}\right\|_{2}\left\|M_{j}\right\|_{2} .
$$

Taking (11) into (9), we get

$$
\begin{aligned}
r_{i j}\left(X_{i}, X_{j}\right) & =\frac{1}{\sqrt{n}} \frac{\left\|M_{i}+M_{j}\right\|_{2}}{\left\|M_{i}\right\|_{\infty}+\left\|M_{j}\right\|_{\infty}} \\
& \leq \frac{1}{\sqrt{n}} \frac{\left\|M_{i}\right\|_{2}+\left\|M_{j}\right\|_{2}}{\left\|M_{i}\right\|_{\infty}+\left\|M_{j}\right\|_{\infty}} \\
& \leq \frac{\sqrt{n}}{\sqrt{n}} \frac{\left\|M_{i}\right\|_{\infty}+\left\|M_{j}\right\|_{\infty}}{\left\|M_{i}\right\|_{\infty}+\left\|M_{j}\right\|_{\infty}} \\
& \leq 1 .
\end{aligned}
$$

(P2) As the relation matrix is $0-1$ matrix, we get $\|M\|_{2}=$ $\sqrt{n}\|M\|_{\infty}$. Then

$$
r_{i i}\left(X_{i}, X_{i}\right)=\frac{1}{\sqrt{n}} \frac{\left\|M_{i}+M_{i}\right\|_{2}}{\left\|M_{i}\right\|_{\infty}+\left\|M_{i}\right\|_{\infty}}=\frac{1}{\sqrt{n}} \frac{2 \sqrt{n}\left\|M_{i}\right\|_{\infty}}{2\left\|M_{i}\right\|_{\infty}}=1 .
$$

\section{The Clustering Algorithm Based on Binary Relation Similarity Measure}

Let $\Phi=\left\{X_{1}, X_{2}, X_{3}, \ldots, X_{p}\right\}$ be a set of vector, let $Q$ be a binary relation on vector $X_{i}$, and let $\delta$ be a threshold value, which is used to determine the similarity between two vectors. If $r_{i j}\left(X_{i}, X_{j}\right) \geq \delta$, that means $X_{i}$ and $X_{j}$ are similar. The clustering algorithm based on binary relation similarity measure can be summarized as follows.

Step 1. We form the relation matrix $M_{i}(i=1,2, \ldots, p)$ based on the binary relation $Q$. Let $U=\left\{M_{1}, M_{2}, \ldots, M_{p}\right\}$ be a set of relation matrix and let $T$ be a temporary set.

Step 2. Let $k$ be number of aggregation, initially, $k=1, i=1$; the threshold value $\delta$ is a real number, such as $0.2,0.3$, and 0.6 .

Step 3. Select $M_{i}$ by orders from the finite set $U, M_{i} \in U$, and then put it into the aggregation $C_{k}$; at the same time, remove $M_{i}$ from $U$; the number of matrixes in $C_{k}$ is $n_{k}=1$.

Step 4. The linear combination of the relation matrix in $C_{k}$ is obtained by

$$
Z_{k}=\sum_{i=1}^{n_{k}} M_{i}
$$

Step 5. If $U$ is nonempty, select the next relation matrix $M_{i}$ $(i=i+1)$ by order from $U$; if $U$ is empty, go to Step 7 .

Step 6. Using (9), the similarity measure between $M_{i}$ and $Z$ can be got. If $r_{i}\left(Z, M_{i}\right) \geq \delta$, assign $M_{i}$ to the aggregation $C_{k}$; at the same time, remove $M_{i}$ from $U, n_{k}=n_{k}+1 ;$ if $r_{i}\left(Z, M_{i}\right)<\delta$, put $M_{i}$ into the temporary set $T$; at the same time, remove $M_{i}$ from $U$. Then, go to Step 4. 
Step 7. If $T$ is nonempty, let $U=T, k=k+1$; go to Step 3, or else, $T$ is empty; go to Step 8.

Step 8. Record the clustering results.

Example 8. Let $\Phi=\left\{X_{1}, X_{2}, X_{3}, X_{4}, X_{5}, X_{6}\right\}$ be a set of vector,

$$
\begin{aligned}
& X_{1}=(0.74,0.71,0.64,0.69,0.93) ; \\
& X_{2}=(0.86,0.03,0.81,0.14,0.28) ; \\
& X_{3}=(0.03,1.00,0.28,0.26,0.44) ; \\
& X_{4}=(0.65,0.01,0.88,0.61,0.14) ; \\
& X_{5}=(0.12,0.61,0.97,0.68,0.86) ; \\
& X_{6}=(0.03,0.72,0.67,0.79,0.94)
\end{aligned}
$$

In the real life, a binary relation can be got through by the analysis of the past data. In an illustrated example, a binary can be given by the decision maker. So in this case, we give the binary relation between the five elements for each vector is " $>$ "; " $>$ " means better or more important, which can be described in mathematic language as $x_{i}-x_{j} \geq 0.4$ ( $i=1,2, \ldots, 5 ; j=1,2, \ldots, 5)$. Using the above clustering algorithm, we get the clustering results as in Table 1; for comparison, we also use the other clustering algorithm in $[22,23]$; the results are shown in Table 1.

In [22], $\mathrm{Xu}$ and Chen defined the consistency index $\rho$ to show the effectiveness of the clustering algorithm; the larger $\rho$ is, the more effective the clustering algorithm is. From Table 1, we can see that the consistency index of our clustering algorithm is larger than the other two clustering algorithms; so our clustering algorithm is more effective.

\section{Multiple Criteria Group Decision Making Method Based on Binary Relation}

In this section, we present a handling method for ITFNs multiple criteria decision making problems. Let $A=\left\{A_{1}\right.$, $\left.A_{2}, \ldots, A_{n}\right\}$ be a set of alternatives, let $C=\left\{C_{1}, C_{2}, \ldots, C_{m}\right\}$ be a set of criteria, and let $B=\left\{B_{1}, B_{2}, \ldots, B_{l}\right\}$ be a set of group members. Assume $Q$ is a binary relation on a finite criteria set $C$. The characteristic of alternative $A_{i}$ for group member $B_{j}$ is represented by the following ITFN:

$$
\begin{aligned}
v_{i}^{j}=\{ & \left\langle C_{1},\left(a_{11 i}^{j}, a_{12 i}^{j}, a_{13 i}^{j}, a_{14 i}^{j}\right),\left(b_{11 i}^{j}, b_{12 i}^{j}, b_{13 i}^{j}, b_{14 i}^{j}\right)\right\rangle, \\
& \left\langle C_{2},\left(a_{21 i}^{j}, a_{22 i}^{j}, a_{23 i}^{j}, a_{24 i}^{j}\right),\left(b_{21 i}^{j}, b_{22 i}^{j}, b_{23 i}^{j}, b_{24 i}^{j}\right)\right\rangle, \ldots, \\
& \left.\left\langle C_{m},\left(a_{m 1 i}^{j}, a_{m 2 i}^{j}, a_{m 3 i}^{j}, a_{m 4 i}^{j}\right),\left(b_{m 1 i}^{j}, b_{m 2 i}^{j}, b_{m 3 i}^{j}, b_{m 4 i}^{j}\right)\right\rangle\right\},
\end{aligned}
$$

for $i=1,2, \ldots, n, j=1,2, \ldots, l$. The decision procedure for the proposed method can be summarized as follows.
TABLE 1: The clustering results for different clustering algorithm.

\begin{tabular}{lccc}
\hline $\begin{array}{l}\text { Different clustering } \\
\text { algorithm }\end{array}$ & $n_{k}$ & $\begin{array}{c}\text { Group } \\
\text { member }\end{array}$ & $\begin{array}{c}\text { The consistency } \\
\text { index } \rho \text { [22] }\end{array}$ \\
\hline $\begin{array}{l}\text { Binary relation clustering } \\
\text { algorithm (BRCA) }\end{array}$ & 5 & $\begin{array}{c}X_{1}, X_{2}, X_{4}, \\
X_{5}, X_{6}\end{array}$ & 0.6450 (BRCA) \\
& 1 & $X_{3}$ & \\
\hline \multirow{2}{*}{ Vector clustering algorithm } & 2 & $X_{1}, X_{2}$ & \\
(VCA) & 1 & $X_{3}$ & 0.3029 (VCA) \\
& 2 & $X_{4}, X_{6}$ & \\
& 1 & $X_{5}$ & \\
Ants-clustering algorithm & 1 & $X_{1}$ & \\
(ACA) & 2 & $X_{2}, X_{3}$ & 0.6442 (ACA) \\
& 1 & $X_{4}$ & \\
& 2 & $X_{5}, X_{6}$ & \\
\hline
\end{tabular}

Step 1. Using (6), we get the expected vector of all group members for each alternative $A_{i}(i=1,2, \ldots, n)$. Let $\Omega_{i}=$ $\left\{E_{i}^{1}, E_{i}^{2}, \ldots, E_{i}^{l}\right\}$ be a set of group member's expected vectors for the alternative $A_{i}$.

Step 2. Using the similarity model (9) and the clustering algorithm in Section 4 , for the alternative $A_{i}$, we get $K(1 \leq$ $K \leq m$ ) aggregations; if $n_{k}$ is the number of expected vector of $k$ th aggregation, then $\sum_{k=1}^{m} n_{k}=m$; the group members in the same aggregation have the relative closeness preference.

Step 3. The expected vector of the aggregation $C_{i}^{k}$ of alternative $A_{i}$ can be obtained by

$$
H_{i}^{k}=\frac{h_{i}^{k}}{\left|h_{i}^{k}\right|},
$$

where $h_{i}^{k}=\sum_{E_{i}^{j} \in C^{k}} E_{i}^{j}$ and $\left|h_{i}^{k}\right|$ is the Euclidean norm of the expected vector of aggregation $C_{i}^{k}$. Then, we calculate the expected vector of the whole group for the alternative $A_{i}$ :

$$
G_{i}=\frac{g_{i}}{\left|g_{i}\right|}
$$

where $g_{i}=\sum_{k=1}^{m}\left(n_{k} / m\right) H_{i}^{k}$ and $\left|g_{i}\right|$ is the length of the expected vector of $g_{i}$.

Step 4. For each alternative $A_{i}$ in $A=\left\{A_{1}, A_{2}, \ldots, A_{n}\right\}$ (where $i=1,2, \ldots, n$ ), using Steps $1-3$, we can get the group expected matrix; that is,

$$
G=\left(G_{1}, G_{2}, \ldots, G_{n}\right)^{T}=\left(\begin{array}{cccc}
g_{1}^{1} & g_{1}^{2} & \cdots & g_{1}^{m} \\
g_{2}^{1} & g_{2}^{2} & \cdots & g_{2}^{m} \\
\cdot & \cdot & \cdot & \cdot \\
g_{n}^{1} & g_{n}^{2} & \cdots & g_{n}^{m}
\end{array}\right)
$$

Step 5. For the weight of the criteria $C_{j}(j=1,2, \ldots, m)$, it is a trapezoidal intuitionistic fuzzy weight $w_{j}=\left\langle\left(c_{1}, c_{2}\right.\right.$, $\left.\left.c_{3}, c_{4}\right),\left(d_{1}, d_{2}, d_{3}, d_{4}\right)\right\rangle(j=1,2, \ldots, n)$. The expected weight 
value $w_{j}(j=1,2, \ldots, n)$ for an intuitionistic trapezoidal fuzzy weight is obtained by (6). Then we normalize the expected weight value $w_{j}(j=1,2, \ldots, n)$ by using the following formula:

$$
w_{j}=\frac{\operatorname{EV}\left(w_{j}\right)}{\sum_{j=1}^{n} \operatorname{EV}\left(w_{j}\right)} .
$$

Step 6. The ranking order of all alternatives $A=\left\{A_{1}, A_{2}\right.$, $\left.\ldots, A_{n}\right\}$ can be obtained as follows:

$$
\begin{aligned}
F=W^{T} G & =\left(w_{1}, w_{2}, \ldots, w_{n}\right)\left(\begin{array}{cccc}
g_{1}^{1} & g_{1}^{2} & \cdots & g_{1}^{m} \\
g_{2}^{1} & g_{2}^{2} & \cdots & g_{2}^{m} \\
\cdot & \cdot & \cdot & \cdot \\
g_{n}^{1} & g_{n}^{2} & \cdots & g_{n}^{m}
\end{array}\right) \\
& =\left(F_{1}, F_{2}, \ldots, F_{n}\right) .
\end{aligned}
$$

According to the maximum principle, we can select the best one by

$$
\text { Best alternative }=\max _{1 \leq i \leq n}\left\{F_{i}\right\}
$$

Then by using (22), we can easily select the best one in the alternative set.

\section{Application and Comparison}

In this section, we use the illustrative example in [19] for a multiple criteria decision making problem, which is a demonstration of the application of the proposed FMCGDM in Section 5.

There is a panel with four possible alternatives to invest the money (adapted from [19]): (1) $A_{1}$ is a car company; (2) $A_{2}$ is a food company; (3) $A_{3}$ is a computer company; (4) $A_{4}$ is a television company. The investment company must take a decision according to the following three criteria: (1) $C_{1}$ is the social benefit; (2) $C_{2}$ is the economical benefit; (3) $C_{3}$ is the environmental impact, where $C_{1}$ and $C_{2}$ are benefit criteria and $C_{3}$ is cost criterion. The four possible alternatives are to be evaluated under the above three criteria by corresponding to the linguistic values of ITFNs for the linguistic terms, as shown in Table 2.

Suppose we invite 3 experts to make judgment on four alternatives. They are expected to give linguistic terms. Then, from Table 2, we convert the linguistic terms to ITFNs, as listed in Table 3.

The decision procedure for the proposed method can be summarized as follows.

Step 1. Using formulation (6), we get the expected value of 3 experts for each alternative, as listed in Table 4.

Step 2. The decision maker gives the binary relation as " $>$," which has been shown in detail in Section 4. The binary relation between criteria is $C_{2}>C_{3}>C_{1}$; “ $>$ " can be described in mathematic as $x_{i}-x_{j} \geq 0.4$. For each alternative
TABLE 2: Nine-member linguistic values of ITFNs.

\begin{tabular}{lc}
\hline Linguistic term & Linguistic values of ITFN \\
\hline Absolutely low & $\langle(0.0001,0.001,0.001,0.001)$, \\
Low & $(0.001,0.001,0.001,0.001)\rangle$ \\
Fairly low & $\langle(0.0,0.1,0.2,0.3),(0.0,0.1,0.2,0.3)\rangle$ \\
Medium & $\langle(0.1,0.2,0.3,0.4),(0.0,0.2,0.3,0.5)\rangle$ \\
Fairly high & $\langle(0.3,0.4,0.5,0.6),(0.2,0.4,0.5,0.7)\rangle$ \\
High & $\langle(0.5,0.6,0.7,0.8),(0.4,0.6,0.7,0.9)\rangle$ \\
Absolutely high & $\langle(0.7,0.8,0.9,1.0),(0.7,0.8,0.9,1.0)\rangle$ \\
\hline
\end{tabular}

$A_{i}(i=1,2,3,4)$, let the threshold value $\delta=0.6$; using similarity model (9) and the clustering method in Section 4, we can get the aggregation $C_{i}^{k}$ and the group members in $C_{i}^{k}$ for each alternative $A_{i}$, as listed in Table 5.

Step 3. Using (17) and (18), we can get the expected vectors of aggregation and group, as listed in Table 5.

Step 4. The weight value of criteria $C_{i}$ is shown in Table 3.

The expected weight values can be obtained by (6). Then we normalize the expected weight value by (20) and get the weight vector $W^{T}=(0.3490,0.3020,0.3490)$.

Step 5. Using the dates in the last row of Table 5, we get the group expected matrix:

$$
G=\left(\begin{array}{llll}
0.5537 & 0.5632 & 0.5649 & 0.7353 \\
0.7665 & 0.6940 & 0.7107 & 0.6199 \\
0.3145 & 0.4407 & 0.4192 & 0.2739
\end{array}\right)
$$

Using (21),

$$
\begin{aligned}
F=W^{T} G= & (0.3490,0.3020,0.3490) \\
& \times\left(\begin{array}{llll}
0.5537 & 0.5632 & 0.5649 & 0.7353 \\
0.7665 & 0.6940 & 0.7107 & 0.6199 \\
0.3145 & 0.4407 & 0.4192 & 0.2739
\end{array}\right) \\
= & (0.5345,0.5599,0.5581,0.5394) .
\end{aligned}
$$

Step 6. Rank the alternatives as follows: $A_{2}>A_{4}>A_{3}>A_{1}$.

Thus, the most desirable alternative is $A_{2}$ in accordance with the above result, which is the same as the C-similarity measure in [19].

Our method's decision results are the same as the Cosine similarity measure's decision results, but our method gets the same results more rapidly than the Cosine similarity measure, especially when the number of the experts is large (for example 100 or 1000). Because an ITFN has 8 elements, each preference vector has 32 elements or more; when the group members are many, the Cosine similarity measure has more complex computations. The decision results of methods in [19] were got by the weighted similarity measure between 
TABLE 3: Preference values of alternative and weights given from 3 experts by ITFN.

\begin{tabular}{|c|c|c|c|}
\hline$A$ & $M$ & $\begin{array}{l}C_{1} \\
C_{3} \\
\end{array}$ & $C_{2}$ \\
\hline \multirow{6}{*}{$A_{1}$} & \multirow{2}{*}{1} & $\langle(0.3,0.4,0.5,0.6),(0.2,0.4,0.5,0.7)\rangle$ & \multirow[t]{2}{*}{$\langle(0.3,0.4,0.5,0.6),(0.2,0.4,0.5,0.7)\rangle$} \\
\hline & & $\langle(0.1,0.2,0.3,0.4),(0.0,0.2,0.3,0.5)\rangle$ & \\
\hline & \multirow{2}{*}{2} & $\langle(0.1,0.2,0.3,0.4),(0.0,0.2,0.3,0.5)\rangle$ & \multirow[t]{2}{*}{$\langle(0.3,0.4,0.5,0.6),(0.2,0.4,0.5,0.7)\rangle$} \\
\hline & & $\langle(0.0,0.1,0.2,0.3),(0.0,0.1,0.2,0.3)\rangle$ & \\
\hline & \multirow{2}{*}{3} & $\langle(0.3,0.4,0.5,0.6),(0.2,0.4,0.5,0.7)\rangle$ & \multirow[t]{2}{*}{$\langle(0.5,0.6,0.7,0.8),(0.4,0.6,0.7,0.9)\rangle$} \\
\hline & & $\langle(0.1,0.2,0.3,0.4),(0.0,0.2,0.3,0.5)\rangle$ & \\
\hline \multirow{6}{*}{$A_{2}$} & \multirow{2}{*}{1} & $\langle(0.5,0.6,0.7,0.8),(0.4,0.6,0.7,0.9)\rangle$ & \multirow[t]{2}{*}{$\langle(0.5,0.6,0.7,0.8),(0.4,0.6,0.7,0.9)\rangle$} \\
\hline & & $\langle(0.3,0.4,0.5,0.6),(0.2,0.4,0.5,0.7)\rangle$ & \\
\hline & \multirow{2}{*}{2} & $\langle(0.5,0.6,0.7,0.8),(0.4,0.6,0.7,0.9)\rangle$ & \multirow[t]{2}{*}{$\langle(0.7,0.8,0.9,1.0),(0.7,0.8,0.9,1.0)\rangle$} \\
\hline & & $\langle(0.3,0.4,0.5,0.6),(0.2,0.4,0.5,0.7)\rangle$ & \\
\hline & \multirow{2}{*}{3} & $\langle(0.3,0.4,0.5,0.6),(0.2,0.4,0.5,0.7)\rangle$ & \multirow[t]{2}{*}{$\langle(0.5,0.6,0.7,0.8),(0.4,0.6,0.7,0.9)\rangle$} \\
\hline & & $\langle(0.3,0.4,0.5,0.6),(0.2,0.4,0.5,0.7)\rangle$ & \\
\hline \multirow{6}{*}{$A_{3}$} & \multirow{2}{*}{1} & $\langle(0.3,0.4,0.5,0.6),(0.2,0.4,0.5,0.7)\rangle$ & \multirow[t]{2}{*}{$\langle(0.5,0.6,0.7,0.8),(0.4,0.6,0.7,0.9)\rangle$} \\
\hline & & $\langle(0.3,0.4,0.5,0.6),(0.2,0.4,0.5,0.7)\rangle$ & \\
\hline & \multirow{2}{*}{2} & $\langle(0.3,0.4,0.5,0.6),(0.2,0.4,0.5,0.7)\rangle$ & \multirow[t]{2}{*}{$\langle(0.5,0.6,0.7,0.8),(0.4,0.6,0.7,0.9)\rangle$} \\
\hline & & $\langle(0.1,0.2,0.3,0.4),(0.0,0.2,0.3,0.5)\rangle$ & \\
\hline & \multirow{2}{*}{3} & $\langle(0.5,0.6,0.7,0.8),(0.4,0.6,0.7,0.9)\rangle$ & \multirow[t]{2}{*}{$\langle(0.5,0.6,0.7,0.8),(0.4,0.6,0.7,0.9)\rangle$} \\
\hline & & $\langle(0.3,0.4,0.5,0.6),(0.2,0.4,0.5,0.7)\rangle$ & \\
\hline \multirow{6}{*}{$A_{4}$} & \multirow{2}{*}{1} & $\langle(0.7,0.8,0.9,1.0),(0.7,0.8,0.9,1.0)\rangle$ & \multirow[t]{2}{*}{$\langle(0.5,0.6,0.7,0.8),(0.4,0.6,0.7,0.9)\rangle$} \\
\hline & & $\langle(0.1,0.2,0.3,0.4),(0.0,0.2,0.3,0.5)\rangle$ & \\
\hline & \multirow{2}{*}{2} & $\langle(0.7,0.8,0.9,1.0),(0.7,0.8,0.9,1.0)\rangle$ & \multirow[t]{2}{*}{$\langle(0.5,0.6,0.7,0.8),(0.4,0.6,0.7,0.9)\rangle$} \\
\hline & & $\langle(0.1,0.2,0.3,0.4),(0.0,0.2,0.3,0.5)\rangle$ & \\
\hline & \multirow{2}{*}{3} & $\langle(0.7,0.8,0.9,1.0),(0.7,0.8,0.9,1.0)\rangle$ & \multirow[t]{2}{*}{$\langle(0.7,0.8,0.9,1.0),(0.7,0.8,0.9,1.0)\rangle$} \\
\hline & & $\langle(0.3,0.4,0.5,0.6),(0.2,0.4,0.5,0.7)\rangle$ & \\
\hline \multirow{6}{*}{ Weights } & \multirow{2}{*}{1} & $\langle(0.3,0.4,0.5,0.6),(0.2,0.4,0.5,0.7)\rangle$ & \multirow[t]{2}{*}{$\langle(0.3,0.4,0.5,0.6),(0.2,0.4,0.5,0.7)\rangle$} \\
\hline & & $\langle(0.3,0.4,0.5,0.6),(0.2,0.4,0.5,0.7)\rangle$ & \\
\hline & \multirow{2}{*}{2} & $\langle(0.3,0.4,0.5,0.6),(0.2,0.4,0.5,0.7)\rangle$ & $\langle(0.3,0.4,0.5,0.6),(0.2,0.4,0.5,0.7)\rangle$ \\
\hline & & $\langle(0.3,0.4,0.5,0.6),(0.2,0.4,0.5,0.7)\rangle$ & \\
\hline & 3 & $\langle(0.5,0.6,0.7,0.8),(0.4,0.6,0.7,0.9)\rangle$ & $\langle(0.3,0.4,0.5,0.6),(0.2,0.4,0.5,0.7)\rangle$ \\
\hline & & $\langle(0.5,0.6,0.7,0.8),(0.4,0.6,0.7,0.9)\rangle$ & \\
\hline
\end{tabular}

TABLE 4: The expected values of ITFNs.

\begin{tabular}{|c|c|c|c|c|c|c|c|c|c|}
\hline Alternatives & Group member & $C_{1}$ & $\mathrm{C}_{2}$ & $C_{3}$ & Alternatives & Group member & $C_{1}$ & $\mathrm{C}_{2}$ & $C_{3}$ \\
\hline$A_{1}$ & 1 & 0.450 & 0.450 & 0.250 & $A_{2}$ & 1 & 0.650 & 0.650 & 0.450 \\
\hline$A_{1}$ & 2 & 0.250 & 0.450 & 0.150 & $A_{2}$ & 2 & 0.650 & 0.850 & 0.450 \\
\hline$A_{1}$ & 3 & 0.450 & 0.650 & 0.250 & $A_{2}$ & 3 & 0.450 & 0.650 & 0.450 \\
\hline$A_{3}$ & 1 & 0.450 & 0.650 & 0.450 & $A_{4}$ & 1 & 0.850 & 0.650 & 0.250 \\
\hline$A_{3}$ & 2 & 0.450 & 0.650 & 0.250 & $A_{4}$ & 2 & 0.850 & 0.650 & 0.250 \\
\hline$A_{3}$ & 3 & 0.650 & 0.650 & 0.450 & $A_{4}$ & 3 & 0.850 & 0.850 & 0.450 \\
\hline
\end{tabular}

TABLE 5: The aggregation of group member based on binary relation, when $\delta=0.6$.

\begin{tabular}{lcccc}
\hline Alternatives & $n_{k}$ & Group member & EV of aggregation & EV of group set \\
\hline$A_{1}$ & 2 & 1,3 & $(0.5974,0.7301,0.3319)$ & \\
$A_{1}$ & 1 & 2 & $(0.4663,0.8393,0.2798)$ & $(0.5537,0.7665,0.3145)$ \\
$A_{2}$ & 1 & 1 & $(0.6351,0.6351,0.4397)$ & \\
$A_{2}$ & 1 & 2 & $(0.5599,0.7322,0.3877)$ & $(0.5632,0.6940,0.4407)$ \\
$A_{2}$ & 1 & 3 & $(0.4947,0.7145,0.4947)$ & $(0.5649,0.7107,0.4192)$ \\
$A_{3}$ & 3 & $1,2,3$ & $(0.5649,0.7107,0.4192)$ & $(0.7353,0.6199,0.2739)$ \\
$A_{4}$ & 3 & $1,2,3$ & $(0.7353,0.6199,0.2739)$ & \\
\hline
\end{tabular}


each alternative and the ideal alternative. In real-world emergence decision making problem, it is difficult to get the ideal alternative; so our method is more applicable in fuzzy multiple criteria large group decision making problem.

\section{Conclusion}

In the complex real-life case, it has complex binary relation between each criterion. Thus, in this paper, we propose a new method for intuitionistic trapezoidal fuzzy multiple criteria group decision problem based on binary relation, in which the preference values for an alternative and the weight values of criteria take the form of ITFNs. The similarity measure between two expected vectors of ITFNs in the vector space based on the binary relation is suggested, and some desirable properties are also proved. Using the similarity measure model, we establish the clustering algorithm for FMCGDM. We also compare our clustering algorithm with the other two clustering algorithms in the reference. Finally, a practical example is given; from the weighted expected vector of group, the ranking order of all alternatives can be determined and the best one(s) can be easily identified as well. The illustrative example shows that the proposed method in this paper is applicable. The comparison between our method and the Cosine similarity measure is given to show our method's effectiveness; the two methods have the same decision results, but our method has simpler computation and gets the same results more rapidly than the Cosine similarity measure method. In the future, we will implement the proposed method into a business decision support system and solve the some real-world problem, such as facility location selection problem, emergence decision making problem, and environment issues.

\section{Conflict of Interests}

The authors declare that there is no conflict of interests regarding the publication of this paper.

\section{Acknowledgments}

The research is supported by the National Natural Science Foundation of China (nos. 11301306, 71171202, and 71371112) and the National Social Foundation of China (no. 12AZD109).

\section{References}

[1] R. E. Bellman and L. A. Zadeh, "Decision-making in a fuzzy environment," Management Science, vol. 17, no. 4, pp. B141-B164, 1970.

[2] X. Chen, M. Liu, and Y. Takahara, "Fuzzy decision making for selection of information system development approach," International Journal of Information and Management Sciences, vol. 9, no. 1, pp. 66-77, 1998.

[3] P. Liu, "A weighted aggregation operators multi-attribute group decision-making method based on interval-valued trapezoidal fuzzy numbers," Expert Systems with Applications, vol. 38, no. 1, pp. 1053-1060, 2011.
[4] Q.-W. Cao and J. Wu, "The extended COWG operators and their application to multiple attributive group decision making problems with interval numbers," Applied Mathematical Modelling, vol. 35, no. 5, pp. 2075-2086, 2011.

[5] P. Liu and F. Jin, "A multi-attribute group decision-making method based on weighted geometric aggregation operators of interval-valued trapezoidal fuzzy numbers," Applied Mathematical Modelling, vol. 36, no. 6, pp. 2498-2509, 2012.

[6] Y. Wang and T. M. S. Elhag, "Fuzzy TOPSIS method based on alpha level sets with an application to bridge risk assessment," Expert Systems with Applications, vol. 31, no. 2, pp. 309-319, 2006.

[7] Y. Tang and C. Chang, "Multicriteria decision-making based on goal programming and fuzzy analytic hierarchy process: an application to capital budgeting problem," Knowledge-Based Systems, vol. 26, pp. 288-293, 2012.

[8] G. W. Wei and Y. Wei, "Model of grey relational analysis for interval multiple attribute decision making with preference information on alternatives," Chinese Journal of Management Science, vol. 16, pp. 158-162, 2008.

[9] S. Chen, "Measure of similarity between interval-valued fuzzy numbers for fuzzy recommendation process based on quadratic-mean operator," Expert Systems with Applications, vol. 38, no. 3, pp. 2386-2394, 2011.

[10] W. L. Hung and M. S. Yang, "Similarity measures of intuitionistic fuzzy sets based on Hausdorff distance," Pattern Recognition Letters, vol. 25, no. 14, pp. 1603-1611, 2004.

[11] L. Dengfeng and C. Chuntian, "New similarity measures of intuitionistic fuzzy sets and application to pattern recognitions," Pattern Recognition Letters, vol. 23, no. 1-3, pp. 221-225, 2002.

[12] H. W. Liu, "New similarity measures between intuitionistic fuzzy sets and between elements," Mathematical and Computer Modelling, vol. 42, no. 1-2, pp. 61-70, 2005.

[13] S. H. Wei and S. M. Chen, "Fuzzy risk analysis based on intervalvalued fuzzy numbers," Expert Systems with Applications, vol. 36, no. 2, pp. 2285-2299, 2009.

[14] L. Zhang, X. Xu, and X. Chen, "A new similarity measure for intuitionistic fuzzy sets and its applications," International Journal of Information and Management Sciences, vol. 23, no. 2, pp. 229-239, 2012.

[15] E. Szmidt and J. Kacprzyk, "A new concept of a similarity measure for intuitionistic fuzzy sets and its use in group decision making," Modeling Decisions for Artificial Intelligence, vol. 3558, pp. 272-282, 2005.

[16] Z. Xu, "Some similarity measures of intuitionistic fuzzy sets and their applications to multiple attribute decision making," Fuzzy Optimization and Decision Making, vol. 6, no. 2, pp. 109-121, 2007.

[17] H. W. Nehi and H. R. Maleki, "Intuitionistic fuzzy numbers and it's applications in fuzzy optimization problem," in Proceedings of the 9th WSEAS International Conference on Systems, pp. 1-5, Athens, Greece, 2005.

[18] J. Ye, "Expected value method for intuitionistic trapezoidal fuzzy multicriteria decision-making problems," Expert Systems with Applications, vol. 38, no. 9, pp. 11730-11734, 2011.

[19] J. Ye, "Multicriteria Group Decision-Making Method Using Vector Similarity Measures For Trapezoidal Intuitionistic Fuzzy Numbers," Group Decision and Negotiation, vol. 21, no. 4, pp. 519-530, 2012.

[20] D. Dubois and H. Prade, "Operations on fuzzy numbers," International Journal of Systems Science, vol. 9, no. 6, pp. 613-626, 1978. 
[21] P. Grzegrorzewski, "The hamming distance between intuitionistic fuzzy sets," in Proceedings of the 10th IFSA World Congress, pp. 35-38, Istanbul, Turkey, 2003.

[22] X. H. Xu and X. H. Chen, "Research on the group clustering method based on vector space," Systems Engineering and Electronics, vol. 27, no. 6, pp. 1034-1037, 2005 (Chinese).

[23] X. H. Xu and Y. F. Fan, "Improved ants-clustering algorithm and its application in multi-attribute large group decision making," Systems Engineering and Electronics, vol. 33, no. 2, pp. 346-349, 2011 (Chinese). 


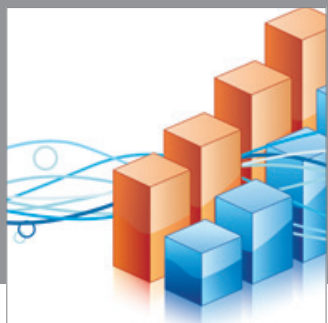

Advances in

Operations Research

mansans

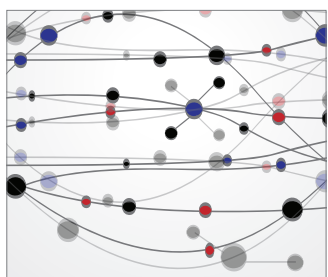

The Scientific World Journal
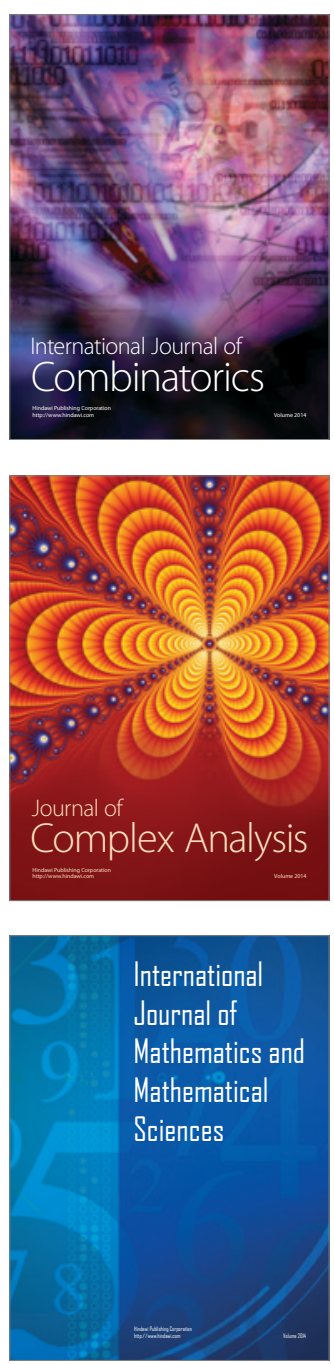
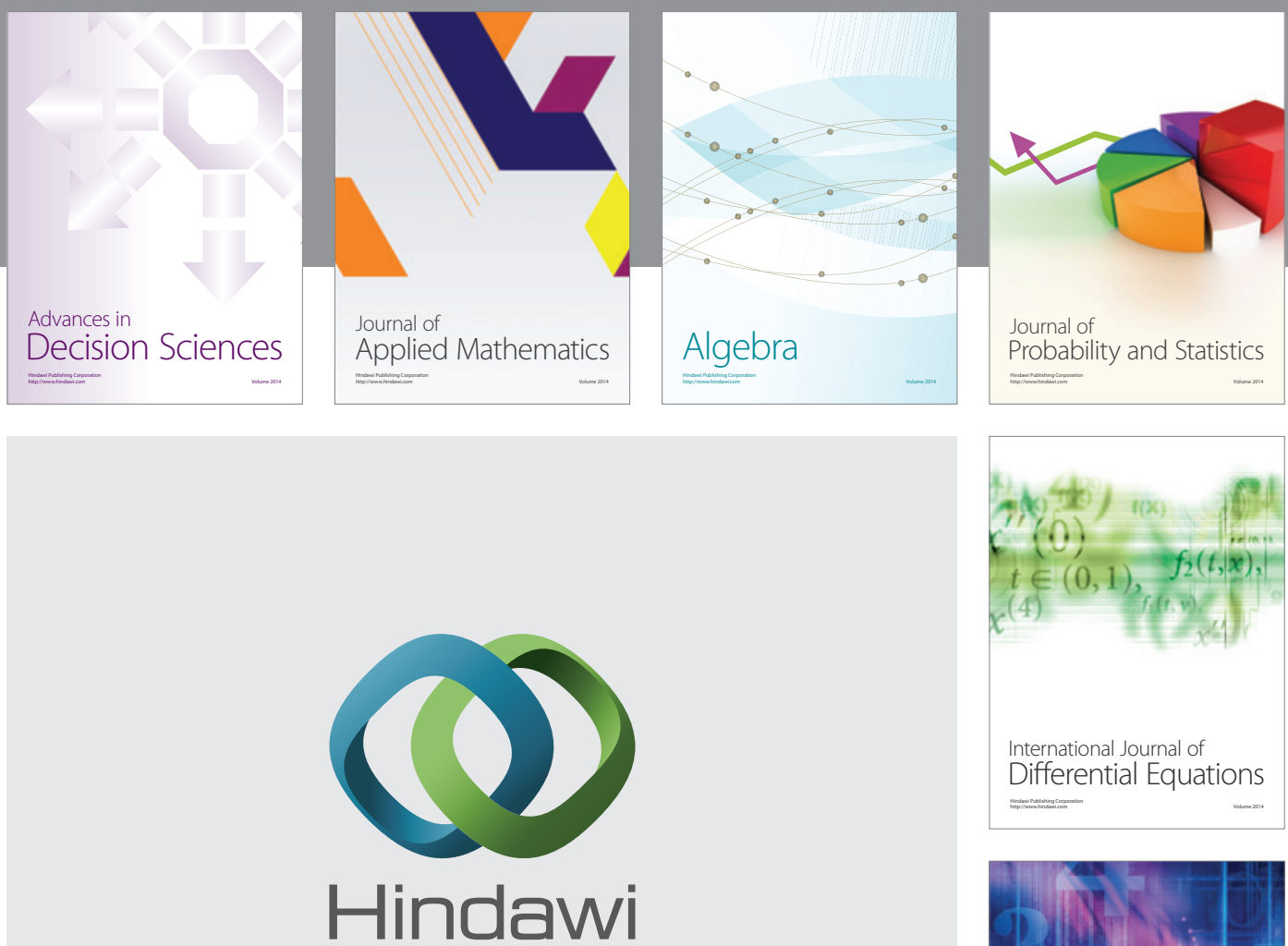

Submit your manuscripts at http://www.hindawi.com
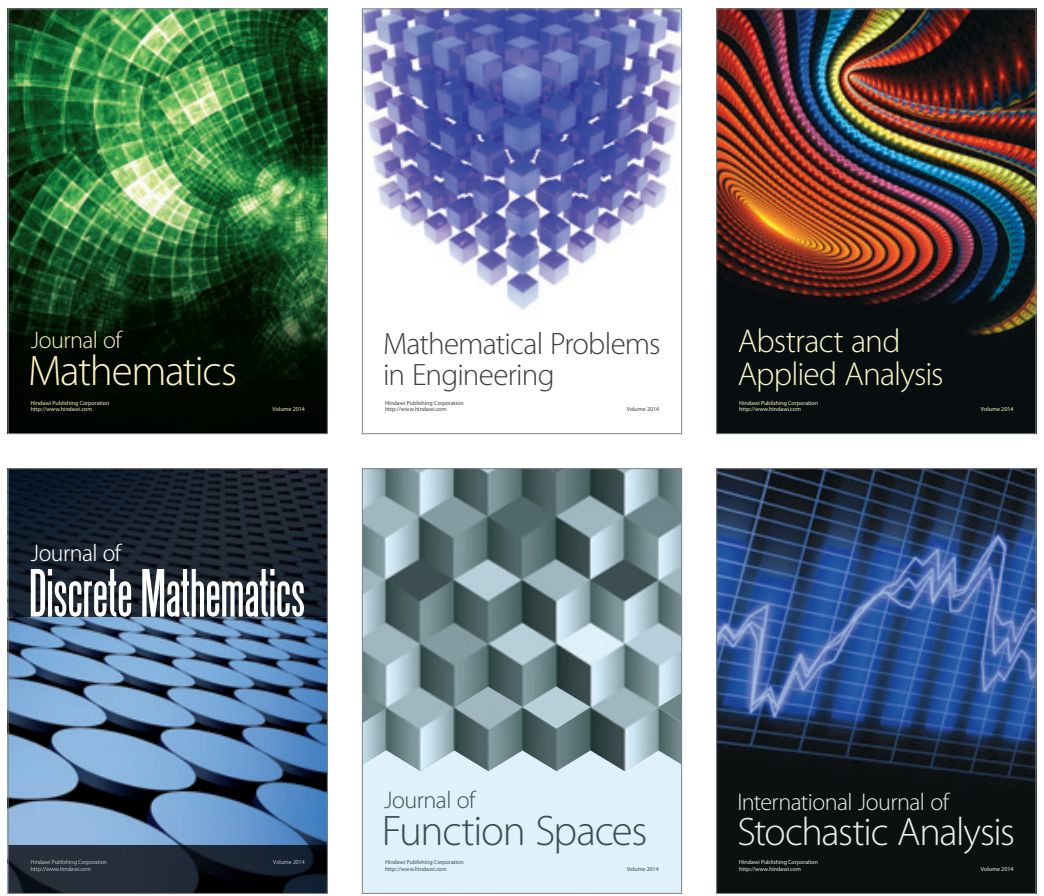

Journal of

Function Spaces

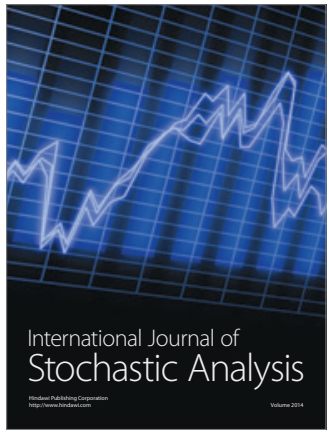

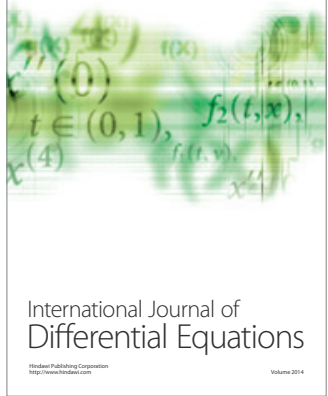
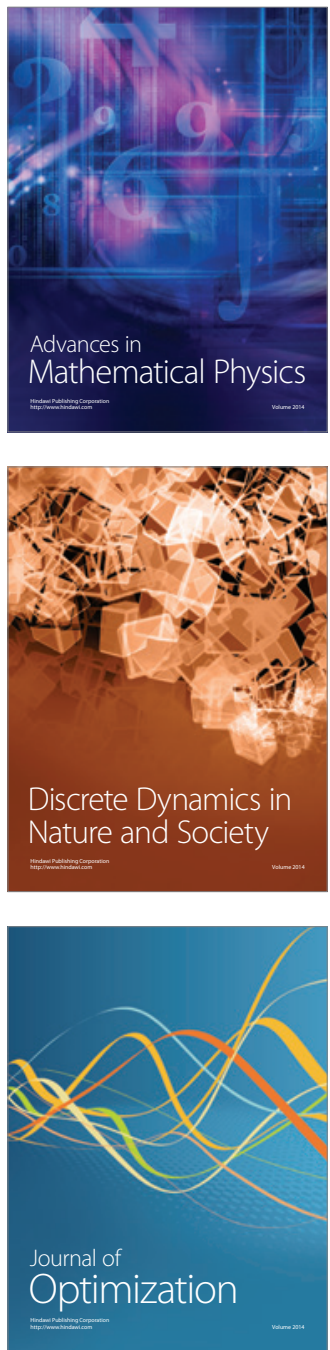